\title{
Die Wahrnehmung der eigenen Professionalität von Chemielehrenden zu unterschiedlichen Zeitpunkten ihrer Berufslaufbahn
}

\author{
Eine empirisch-quantitative Studie
}

\section{Alexander Lengauer ${ }^{1}$}

https://doi.org/10.53349/resource.2021.i16.a1006

\section{Zusammenfassung}

In diesem Beitrag werden nach einer kurzen Einführung in das EPIK-Modell und das Laufbahnmodell von Huberman die Ergebnisse einer Studie präsentiert, die untersucht, ob sich das subjektiv wahrgenommene Professionsempfinden von Chemielehrenden der Sekundarstufe im Laufe der Berufsausübung verändert. Dabei wurde primär untersucht, inwieweit die Faktoren Dienstalter, Ausbildungsvariante, Fortbildungstätigkeit oder Geschlecht einen Einfluss auf die selbst wahrgenommenen Kompetenzen haben. Als quantitative Studie konzipiert erfolgte eine Befragung der Mitglieder des Verbands der Chemielehrer/innen Österreichs (VCÖ) per Online-Fragebogen mit rund 50 Items, wobei von knapp 200 Teilnehmer*innen Fragen zur gefühlten Professionalität beantwortet wurden. Im Zuge der deskriptiven sowie inferenzstatistischen Auswertung (einfaktorielle MANOVA sowie t-Test nach Pearson) konnte gezeigt werden, dass sowohl das Dienstalter als auch die Fortbildungstätigkeit einen statistisch signifikanten Einfluss auf die selbstwahrgenommene Professionalität in einigen Bereichen haben, wohingegen die absolvierte Ausbildung und das Geschlecht von geringer Bedeutung sind. Die Ergebnisse legen den Schluss nahe, dass eine Lehrperson zu keinem Zeitpunkt der Berufslaufbahn tatsächlich „fertig“ ist, sondern die Wahrnehmung als professionell arbeitende Pädagogin*arbeitender Pädagoge ständigen Veränderungen unterworfen ist.

\section{Keywords:}

Pädagogische Professionalität

Chemieunterricht

Berufslaufbahn von Lehrenden

\section{Einleitung}

Der zentrale Bestandteil im Diskurs zur Verbesserung von Unterrichtsqualität sind die im Klassenzimmer stehenden Pädagog*innen. Unter dem Schlagwort Professionalisierung wird dabei regelmäßig eine Reihe an Maßnahmen diskutiert, die Lehrenden das pädagogische Rüstzeug verleihen, um in ihrer Profession erfolgreich zu sein (u. a. die Konzipierung von Aus-, Fort- und Weiterbildung). Professionalität wird dabei häufig als professionelles Handeln definiert. Ein Problem liegt jedoch bislang darin, dass sich viele Lehrer*innen selbst kaum als professionell Handelnde wahrnehmen.

Die höhere Sinnebene [...], die eine Fachsprache mit entsprechenden wissenschaftlichen Erklärungen, Theorien und empirisch gesicherten Wissensbeständen enthält, in der die Differenz zu Alltagstheorien und zum Laien zum Ausdruck kommt, ist bei Lehrern und Lehrerinnen hinsichtlich ihrer pädagogischen Tätigkeit kaum entwickelt. (Bastian \& Helsper, 2000, S. 170)

\footnotetext{
${ }^{1}$ Pädagogische Hochschule Wien, Grenzackerstraße 18, 1100 Wien.

E-Mail: alexander.lengauer@phwien.ac.at
} 
Dabei geht es weniger um Professionalität, die sich aus dem Fachwissen ableitet. Vielmehr stehen unter anderem das Begründen gesetzter pädagogischer Handlungen, die Diskursfähigkeit, die Fähigkeit zur kritischen Selbstreflexion, das Definieren von Zielen sowie der persönliche Wunsch nach einer langfristigen Verbesserung der eigenen Fertigkeiten und Fähigkeiten im Zentrum. Ein Ansatz, Professionalität greif- und messbar zu machen, ist das EPIK-Modell von Schratz et al. (2007a; 2007b), welches diese in fünf Teildisziplinen, sogenannte Domänen, unterteilt und eine strukturierte Möglichkeit darstellt, Professionalität empirisch zu untersuchen.

Die Professionalität von Lehrpersonen ist jedoch kein starres, unveränderliches Konstrukt. Vielmehr unterliegt sie für jede Lehrperson einer permanenten Veränderung. Die berufsbiographischerziehungswissenschaftliche Forschung beschäftigt sich seit Jahrzehnten mit dem Verlauf von Lehrerlaufbahnen. Konzentrierte sich die Forschung lange Zeit auf die Phase des Berufseinstiegs, verschob sich in jüngerer Vergangenheit das Interesse in Richtung gesamter Berufslaufbahn. Terhart (2001) begründet dies damit, „dass Lehrer im Laufe des gesamten Berufslebens Änderungen hinsichtlich der Haltung zum Beruf, hinsichtlich ihrer Schwerpunktsetzungen, hinsichtlich ihrer Selbstdeutung etc. erfahren" (S. 60). In einer Reihe von Berufslaufbahnmodellen wie beispielsweise jenen von Fuller \& Brown (1975, zitiert nach Messner \& Reusser, 2000, und nach Terhart et al., 1994) sowie Huberman (1991) wird diesem Umstand Rechnung getragen.

Diesen Modellen ist jedoch ihre fehlende Fachspezifität gemein. Es scheint lediglich „die Lehrenden“ zu geben. Es gibt selbstverständlich eine unvorstellbar große Zahl an didaktischen Publikationen für jedes erdenkliche Fach. Inwieweit sich jedoch die selbst empfundene Professionalität in einem bestimmten Unterrichtsgegenstand mit allen seinen inhaltlichen wie fachdidaktischen Spezifika im Laufe der Berufsausübung verändert, wird von diesen Modellen nicht berücksichtigt. Ein aus Sicht des Autors in vielerlei Hinsicht besonderes Fach ist die Chemie. Nicht nur bilden Lehrende dieses Fachs an ihren Schulstandorten eine vergleichsweise kleine, in vielen Stundentafeln stiefmütterlich behandelte Gruppe. Das Unterrichtsfach Chemie stellt zusätzlich wie auch beispielsweise sportlich oder kreativ orientierte Fächer spezifische Herausforderungen an die Lehrenden (Lehrer- und Schülerexperimente, forschendes Lernen, hoher Abstraktionsgrad der Inhalte, Fachsprache).

Ebenso wenig berücksichtigen Laufbahnmodelle die unterschiedlichen Ausbildungswege, welche man unter anderem in Österreich durchläuft, um als Lehrperson arbeiten zu können. Dabei stellt sich die Frage, ob sich Lehrende, welche das universitäre Lehramtsstudium absolviert haben, hinsichtlich ihres Professionsempfindens von jenen unterscheiden, die eine dreijährige $\mathrm{PH}$ - oder Pädak-Ausbildung abgeschlossen haben.

Die vorliegende Studie nahm sich zum Vorsatz, diese Forschungslücke zu füllen. Vor dem Hintergrund verschiedener Professionstheorien und verschiedener Berufslaufbahnmodelle wurde untersucht, inwieweit sich das subjektive Wahrnehmen als Professionalist*innen bei Chemielehrenden im Laufe ihrer Karriere ändert. Primär ging es dabei um den Faktor Dienstjahre, wodurch die Frage beantwortet werden sollte, ob mit Berufserfahrung automatisch eine Selbstwahrnehmung als Profi einhergeht oder möglicherweise gar ein gegenteiliger Effekt eintritt und Lehrende gegen Ende der Karriere ihre Fertigkeiten niedriger einschätzen, als es dienstjüngere Lehrpersonen tun. Weiters sollte die Frage beantwortet werden, inwieweit sich aktive Fortbildungstätigkeit auf die subjektiv wahrgenommene Professionalität auswirkt. Der Vergleich zwischen den Ausbildungsvarianten ist insofern praxisrelevant, als erst unlängst die PädagogInnenbildung NEU implementiert wurde, welche primär die Handschrift des bisherigen universitären Lehramtsstudiums trägt. Würden sich aber Pädak- oder PH-Absolvent*innen als professioneller wahrnehmen, wäre dies durchaus relevant für die Gestaltung der zukünftigen Curricula. Abschließend sollte auch untersucht werden, ob sich weibliche und männliche Lehrpersonen unterschiedlich professionell wahrnehmen.

Die Arbeit wurde als empirisch-quantitative Studie konzipiert. Auf Basis der verwendeten Literatur wurde ein Fragebogen entworfen, in welchem Chemielehrende ihre eigenen fachspezifischen Fertigkeiten gemäß den Domänen des EPIK-Modells einschätzen sollten. Die Antworten wurden im Anschluss daran mit gängigen deskriptiven und inferenzstatistischen Verfahren ausgewertet.

\section{Theoretische Vorannahmen}

Wenngleich sich in der professionstheoretischen Literatur eine Reihe von Definitionen finden lässt, gibt es kein einheitliches, allgemeingültiges Verständnis dessen, was unter Professionalität genau zu verstehen ist (Stahl, 1995, S. 39). Ein Ansatz, diesen Begriff dennoch greif- und messbar zu machen, ist das EPIK-Modell. Um die Berufslaufbahn von Lehrpersonen zu quantifizieren, wurde das Laufbahnmodell von Huberman (1991) ausgewählt. Beide Ansätze werden im Folgenden kurz skizziert. 


\subsection{Das EPIK Modell}

Im Jahr 2005 wurde vom damaligen Bundesministerium für Bildung, Wissenschaft und Kultur (BM:BWK) eine Arbeitsgruppe unter der Leitung von Michael Schratz eingesetzt, um ein neues, weitreichendes Professionalisierungskonzept für Lehrende zu entwickeln. Das Ergebnis dieses Projekts firmiert heute unter dem Begriff EPIK (Entwicklung von Professionalität im internationalen Kontext) und bildet die pädagogische Professionalität mittels fünf Kompetenzfelder, sogenannte Domänen, ab. Diese Teilbereiche schließen fachspezifisches und implizites Wissen ebenso ein wie Haltungen. Bei den einzelnen Domänen geht man davon aus, dass Strukturen vorhanden sind, in denen sich diese Kompetenzen entwickeln können. Neben den Kompetenzen einer Lehrkraft schließen die Domänen aber auch den professionellen Habitus mit ein. Weiters zeichneten sich Domänen dadurch aus, dass sie nicht abgeschlossen seien. Sie böten „zahlreiche Anknüpfungspunkte für Überlegungen zur Schulentwicklung, zur Weiterentwicklung der Aus- und Fortbildung der Lehrerinnen und Lehrer" (Paseka et al., 2011, S. 24), seien alle miteinander verknüpft und einer permanenten Entwicklung unterworfen. Ferner seien sie unabhängig von Schultyp und Unterrichtsfach. Die fünf EPIK-Domänen heißen Reflexions- und Diskursfähigkeit, Professionsbewusstsein, Differenzfähigkeit, Kollegialität sowie Personal Mastery. Als verbindendes Element und in Anlehnung an Senge (2006) führt die Arbeitsgruppe die "Sechste Disziplin" ein. In dieser werden die einzelnen Domänen in Beziehung gesetzt und kontextspezifische Aspekte wie Schultyp und Fachdidaktik einbezogen. Da diese Punkte in allen fünf Domänen wirksam sind und daher nicht herausgelöst betrachtet werden sollen, verzichten die Autor*innen darauf, sie als eigene Domäne darzustellen. Vielmehr entsprechen die einzelnen Domänen bildlich gesprochen Puzzleteilen, die durch die sechste Disziplin zusammengehalten würden (Paseka et al., 2011).

Die Domäne Reflexions- und Diskursfähigkeit beinhaltet unter anderem die Fähigkeit und Bereitschaft, das eigene Handeln immer wieder neu kritisch zu hinterfragen sowie dabei mittels Fachsprache in einen Diskurs mit anderen Handelnden zu gehen (Paseka et al., 2011, S. 28). Unter Professionsbewusstsein wird die Fähigkeit verstanden, sich als Expert*in wahrzunehmen und sich selbst als Teil einer Profession zum Thema des Diskurses zu machen. In diesem Bereich kompetente Lehrpersonen nehmen ihr Wissen und Können bewusst wahr. Sie sind vertraut mit dem Ablauf von Lernprozessen und sind in der Lage, Methoden situationsspezifisch einzusetzen und auch zu argumentieren. Zu dieser Domäne gehören auch die realistische Einschätzung des persönlichen Wirkungsbereichs, Offenheit gegenüber Veränderungen und eine ständige Fort- und Weiterbildung (Uhl, 2017; Paseka et al., 2011). Die dritte Domäne des EPIK-Modells, Kooperation und Kollegialität bildet die Steigerung von Produktivität durch Zusammenarbeit ab. Begründet wird dies damit, dass in einer von zunehmender Komplexität gezeichneten Berufsrealität das Gelingen von Schule und Unterricht von der Kooperation der im Feld handelnden Professionalist*innen abhängig ist. Ein Mehr an Kooperation wirke sich in weiter Folge unter anderem auf die Berufszufriedenheit aus. Begründet wird dies von Uhl (2017) mit dem Umstand, dass der Mensch per se ein kooperatives Wesen sei. Zusammenarbeit führe in der Regel zu einer größeren Erfolgswahrscheinlichkeit, was wiederum die Motivation und Berufszufriedenheit erhöhe. Der Kompetenzbereich Differenzfähigkeit hat die täglichen Herausforderungen von Lehrenden hinsichtlich der akademischen, sozialen und kulturellen Diversität der zu unterrichtenden Kinder und Jugendlichen zum Inhalt. „Differenzfähigkeit umfasst die Kompetenz, den unterschiedlichen Geschwindigkeiten und Lernstilen sowie der Bandbreite an verschiedenen Interessen, Leistungs- und Lernbereitschaften von Schülerinnen und Schülern mit Zugewandtheit und Know-how einfühlsam zu begegnen" (Schratz \& Schrittesser, 2011, S. 195). In diesem Bereich kompetent handelnde Lehrpersonen würden somit Unterschiede erkennen, diese sowohl als Herausforderung als auch als Chance verstehen und eine Reihe von Strategien und Methoden anzuwenden wissen, um den Unterschieden in ihrer Klasse gerecht zu werden. Dazu zählten beispielsweise verschiedene Vorgehensweisen bei Lernproblemen oder Kommunikationsschwierigkeiten oder die Gestaltung von Materialien, die eine innere Differenzierung ermöglichten. Die Personal Mastery bildet die fünfte Domäne. Ihr zentrales Merkmal ist dabei, dass Lehrende nicht nur über Wissen (know what) und Können (know how) verfügen, sondern beides auch auf Basis ihrer Professionalität zum richtigen Zeitpunkt einzusetzen vermögen. Dabei bildet das klassische Fachwissen nur das Fundament der Domäne. Ferner sei die formale Ausbildung nicht der zentrale Aspekt der persönlichen Könnerschaft. Vielmehr sei diese das Ergebnis eines „subjektbezogenen Bildungsprozesses, der dazu anleitet, den eigenen Weg zu finden" (Uhl, 2017, S. 69). Wichtig ist dabei, dass sich die Lehrperson nicht lediglich als Expert*in für pädagogisches Wissen oder Handeln sieht, sondern sich als Kenner*in ihrer*seiner selbst wahrnimmt. Nur wer in der Lage ist, sein eigenes Handeln einer permanenten kritischen Prüfung zu unterziehen, vermag seine persönliche Könnerschaft zu verbessern. In dieser Hinsicht professionelle Lehrpersonen verstehen die eigene Persönlichkeit als etwas Unvollendetes. Fehler werden dabei als Schritte zur weiteren 
Professionalisierung verstanden. Personal Mastery endet daher nicht mit dem Abschluss der Ausbildung, sondern ist vielmehr das Ergebnis einer langfristigen Selbstreflexion.

\subsection{Das Laufbahnmodell nach Huberman}

Entgegen der unter Lehrenden wie auch Lehramtsstudierenden weitverbreiteten Meinung ist man mit dem Abschluss des Studiums nicht fertig: „Die gedankliche Formel von ,Studium+Praktika+Referendariat = fertiger Lehrer' muss aufgegeben werden. Es gibt keine ,fertigen Lehrer'“ (Herrmann \& Hertramph, 2000, S. 187). Vielmehr beginne die Entwicklung mit dem ersten Seminar und ende mit dem Ausscheiden aus dem Dienst. Eine Einschätzung, welche auch von Behr (2017) geteilt wird. Die Entwicklung als Lehrperson sei als lebenslanger Prozess zu verstehen. Nach der Phase der Ausbildung und jener des Berufseinstiegs bilde die dritte Phase, welche von ständiger Fort- und Weiterbildung gekennzeichnet sei, einen wichtigen Abschnitt der Berufsbiographie. Unter dem Schirmbegriff lebenslanges Lernen werden dabei Fort- und Weiterbildungsmaßnahmen zusammengefasst, welche Lehrpersonen bis zu ihrem Karriereende begleiten (Dammerer, 2020).

In Publikationen aus dem Feld der berufsbiographischen Forschung wie Hericks (2006) zeigt sich, dass vor allem der Übergang von formaler Ausbildung zum Eintritt in die berufliche Praxis besonders häufig untersucht wird. Aus den verhältnismäßig wenigen empirisch gut abgesicherten Laufbahnmodellen, welche die gesamte Berufsbiographie abdecken, sind vor allem Fuller \& Brown (1975), Dreyfus \& Dreyfus (1988), Sikes et al. (1991) sowie Huberman (1991) zu erwähnen.

Während Fuller \& Brown (1975) die Laufbahn von Lehrenden in drei Stufen teilen und dabei auf eine klare Zuteilung der drei Stufen zu Dienstjahren oder biologischem Alter der Lehrenden verzichten, weist Huberman (1991) seinen fünf Stufen der beruflichen Entwicklung eine genaue Zahl an Dienstjahren zu. Sikes et al. (1991) gehen hingegen vom biologischen Alter der Lehrenden, unabhängig vom Dienstalter, aus. Weder Fuller \& Brown (1975), Sikes et al. (1991) noch Dreyfus \& Dreyfus (1988) gehen auf unterschiedliche Karriereverläufe ein. Vielmehr sind diese Modelle sehr linear angelegt und zeigen nach Ansicht des Autors deutliche Schwächen. So gehen Dreyfus \& Dreyfus (1988) davon aus, dass Talent und Erfahrung ausreichen, um sich zum Experten*zur Expertin entwickeln zu können. Das Modell nach Huberman (1991) hingegen geht auf die sich unterscheidenden Karriereverläufe ein. Hinzu kommt, dass dieses Modell empirisch am umfangreichsten untersucht wurde und dabei sowohl qualitative als auch quantitative Methoden zum Einsatz kamen (Dammerer, 2020). Somit ist Hubermans Modell am besten geeignet, um als theoretische Grundlage für einen Teil dieser empirischen Untersuchung zu dienen.

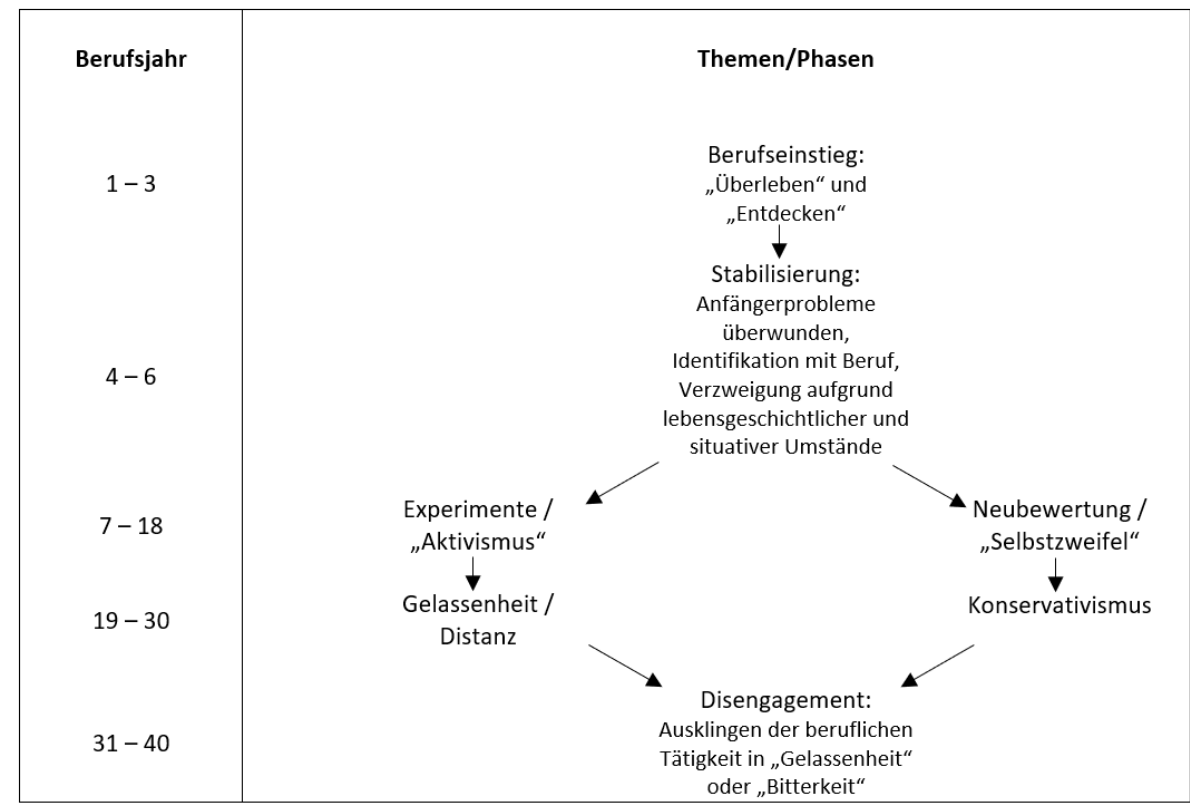

Abbildung 1: Das Phasenmodell nach Huberman (nach 1991, S. 249)

Zu Beginn der Karriere gibt es Huberman (1991) zufolge noch wenige Unterschiede zwischen den einzelnen Lehrpersonen. Die Phase des Berufseinstiegs dauert während der ersten drei Dienstjahre an und ist vom 
„Überleben“ und „Entdecken“ gekennzeichnet. Das Hineinfinden in die neue Rolle als Lehrperson fällt in diese Phase. Huberman (1991) unterscheidet in seinen Erläuterungen dabei jedoch zwischen gelungenen und misslungenen Berufseinstiegen: „Ein leichter Start bedeutet positive Beziehungen zu Schülern, leicht zu handhabende Schüler, ein Gefühl von pädagogischem Geschick sowie Enthusiasmus. Ein schlechter Start bedeutet Rollenüberlastung und Angst, schwierige Schüler, hoher Zeitaufwand, dichte Kontrolle durch Ausbilder sowie Isolation innerhalb der Schule" (S. 253). Auch das berufliche Selbstvertrauen bzw. die Überzeugung, bereits zu diesem frühen Zeitpunkt über pädagogische Fertigkeiten zu verfügen, wirkt sich auf das Erleben des Berufseinstiegs aus. In diesem Modell besteht somit auch die Möglichkeit der völligen Überforderung am Beginn der Laufbahn (Klusmann et al., 2012). An dieser Stelle muss jedoch erwähnt werden, dass ein schlechter Berufseinstieg nicht notwendigerweise einen negativen berufsbiographischen Verlauf zur Folge haben muss. Vielmehr hat eine Lehrperson an verschiedenen Zeitpunkten der Berufslaufbahn die Möglichkeit, sich infolge ausgiebiger Reflexion als Lehrperson neu zu erfinden (Dammerer \& Schwab, 2019).

Im Anschluss an den Einstieg erfolgt die dreijährige Phase der "Stabilisierung". In diesem Abschnitt beginnen sich die jungen Lehrpersonen im Klassenzimmer wohlzufühlen und sich vermehrt mit der Gestaltung von Unterrichtseinheiten zu beschäftigen. „Die Anfängerprobleme sind überwunden, man beherrscht die grundlegenden Unterrichtstechniken und identifiziert sich mit dem Lehrersein“ (Messner \& Reusser, 2000, S. 160). Ferner emanzipiert sich die Lehrperson in dieser Phase in einem Ausmaß, das eigene Wege erlaubt. Auch Kraler (2008) betont, dass sich in dieser Phase des Modells „das Schwergewicht zunehmend zum Entdecken verlagert" (S. 5), und Konflikte mit erfahreneren Lehrpersonen könnten aufgrund des erlangten Selbstbewusstseins in Kombination mit neuen Zugängen häufiger auftreten.

Ab der dritten Phase, welche sich im Bereich des 7. bis 18. Dienstjahres ereignet, differenziert Huberman (1991) zwischen verschiedenen Laufbahnentwicklungsmöglichkeiten. Ein Teil der Lehrpersonen wählt an diesem Punkt die Phase des Experimentierens. Dabei geht es, nachdem das grundsätzliche Unterrichtshandwerk erlernt wurde, um den Versuch, globale Veränderungen herbeizuführen. Dammerer (2020) folgend kann sich in dieser Phase der Wunsch manifestieren, „,in der hierarchischen Struktur der Schule als Institution aufzusteigen“ (S. 65), also beispielsweise eine Leitungsposition anzustreben. Lehrende, welche diesen Weg einschlagen, empfinden die angeeignete Routine teilweise mehr als Last denn als Vorteil. Der Gedanke, nun lebenslang in der Laufbahn als Lehrperson festzustecken, bereitet innen Sorge. Alternativ kann eine Lehrperson in der dritten Phase aber auch den Weg der Neubewertung oder des Selbstzweifels einschlagen. Diese Lehrenden sind von der einsetzenden Routine derart gelangweilt, dass sie daran zweifeln, den richtigen Beruf gewählt zu haben. Die Ursache dafür sei vor allem in „der geringen ,Qualität des Arbeitsplatzes““ zu suchen bzw. bei „Aspekten, die mit Unterrichtsanleitung, kollegialem Austausch und den Gelegenheiten für besondere Weiterbildung zu tun haben“ (Huberman, 1991, S. 257).

Wie die vierte Phase aussieht, hängt von der vorhergehenden Phase ab. War die dritte Phase vom Experimentieren gekennzeichnet, beschreitet die Lehrperson anschließend den Weg der Gelassenheit bzw. der Distanz. Dies äußert sich beispielsweise dadurch, dass sich Lehrende in dieser Phase andere Betätigungsfelder in oder neben der Schule suchen. Dem gegenüber stehen jene Lehrpersonen, welche die konservative Phase durchlaufen. Dies trifft häufig Lehrende, welche in der vorhergehenden Phase Zweifel an ihrer Wirksamkeit im Zuge ihrer Berufsausübung entwickelt haben. Salopp formuliert verklären diese Lehrer*innen die schulische Vergangenheit. Aktuelle Entwicklungen werden implizit dafür verantwortlich gemacht, dass man als Lehrperson heute nicht mehr erfolgreich sein könne: „Die Schüler verfügten über weniger Vorwissen und sind weniger motiviert; der Lehrer erstickt in Verwaltungsarbeit: hirnlose Bürokraten hätten vor dem Zeitgeist kapituliert“ (Huberman, 1991, S. 258).

In der letzten Phase kommt es entweder zu Gelassenheit oder Bitterkeit. Während Letzteres von Frustration und dem Herbeisehnen eines Schlussstrichs gekennzeichnet ist, ist eine gelassene Lehrperson beeinflusst von gelöstem Realismus und häufig von der Frage geleitet, „ob das System tatsächlich veränderbar ist und im Gegenteil nicht vielmehr Disengagement sogar befördere" (Huberman, 1991, S. 263).

Der große Vorteil dieses Modells ist neben der Ausrichtung am beruflichen statt dem biologischen Alter die Berücksichtigung von unterschiedlich verlaufenden Entwicklungen im Laufe der Berufsausübung. Das Modell ist dadurch weniger idealtypisch und realitätsnäher. So kann sich nach den ersten sechs Jahren eine Lehrperson entweder zu Aktivismus bzw. Engagement oder zu Selbstzweifeln bzw. Disengagement entwickeln - abhängig unter anderem vom schulischen Umfeld. Bis hin zum Ruhestand kann dies Gelassenheit oder Zynismus zur Folge haben. Kritisch sehen Messner \& Reusser (2000) den Umstand, dass trotz der empirischen Absicherung des Modells es „nicht möglich zu sein scheint, diese Entwicklung anhand von Prädiktoren wirklich vorherzusagen“ (S. 160). Vielmehr seien erst ab der dritten Phase erste weitere Prognosen hinsichtlich der Entwicklung zulässig. 
Dennoch gilt dieses Modell als empirisch gut abgesichert. Wenngleich die dazugehörige Studie aus dem Jahr 1989 stammt, hat sie laut Schmidt et al. (2016) nichts an ihrer Aktualität verloren.

\subsection{Chemielehrende}

Chemielehrende bilden an Schulen eine besondere Gruppe. Zu der meist sehr überschaubaren Anzahl an Fachlehrpersonen am Standort kommen wenige Wochenstunden in den einzelnen Klassen hinzu. Der Wunsch, dieses Fach fachfremd zu unterrichten, hält sich häufig in engen Grenzen. Die Ursache könnte darin liegen, dass das Fach als „schwer" oder Versuche gar als „gefährlich“ wahrgenommen werden. Wenngleich das Fach Wilhelm, Rehm \& Reinhardt (2018) folgend in vielen Bereichen den gleichen didaktischen Prinzipien folgt wie andere Bestandteile des Fächerkanons (Methodenvielfalt, inhaltliche Klarheit, Strukturierung des Unterrichts), gibt es dennoch fachspezifische Kompetenzen sowie im Vergleich zur Alltagssprache größere Abstraktionen hinsichtlich der Unterrichtssprache, deretwegen das Fach Chemie eine Sonderstellung einnimmt. So bezeichnen Eilks \& Marks (2018) das Experiment als zentrales Element von wirksamem Chemieunterricht, wodurch sich die Chemie abgesehen von der Physik bzw. mit Abstrichen von der Biologie von allen anderen Fächern in der Sekundarstufe unterscheidet. Rehm (2018) bezieht sich dabei auf das Johnstone-Dreieck aus dem Jahr 1991, wonach chemischer Unterricht durch „die Ebene der Phänomene (das Beobachtbare), die sub-mikroskopische Ebene (die atomare Denkebene) und die symbolische Ebene (die Formelsprache, Modelle etc.)“ (Rehm, 2018, S. 18) eine Sonderstellung einnimmt. Das Einbeziehen von chemiespezifischen Schülervorstellungen sowie der didaktisch sinnvolle Einsatz von Experimenten in verschiedenen Sozialformen runden die Fachspezifität ab. Aufgrund dieser Besonderheiten lässt sich eine gezielte Untersuchung dieser Fachlehrenden begründen.

\section{Methodische Vorgehensweise}

Um die Forschungsfrage zu beantworten, wurde eine quantitative Studie konzipiert. Dadurch sollte eine objektive Messung sowie eine numerische Darstellung der professionellen Selbstwahrnehmung von Lehrenden ermöglicht werden. Im Anschluss an die Fragebogengestaltung und Datenerhebung erfolgte die deskriptive sowie inferenzstatistische Auswertung mit SPSS 26.

\subsection{Untersuchungsdesign}

Im Rahmen des Literaturstudiums wurde eine Reihe von wissenschaftlich fundierten Fragebögen von Autor*innen eingeholt. Die an der TU Braunschweig von Hilfert-Rüppell et al. (2012) durchgeführte Untersuchung zum Professionalisierungsprozess in den naturwissenschaftlichen Fächern der Lehramtsstudiengänge untersuchte die selbst wahrgenommene Professionalisierung von Lehramtsstudierenden mithilfe eines quantitativen Fragebogens. Aus diesem Fragebogen wurden 41 ordinalskalierten Items (exklusive demographischer Items wie Geschlecht und Dienstalter) ausgewählt, bei denen die zu Befragenden ihre eigene Professionalität einschätzen und mittels einer fünfstufigen Ratingskala beurteilen sollten. Die Antwortmöglichkeiten reichten dabei von sehr hoch bis nicht vorhanden. Ergänzt wurden sie um einzelne Fragen, welche der Umfrage „Schulrealitätsbezug“ des Departments „Qualität“ der Pädagogischen Hochschule Niederösterreich entnommen wurden (persönliche Kommunikation mit Mag. Ulrike Koglbauer, 12. Jänner 2020). In Tabelle 1 werden einige Beispielitems aus den verschiedenen Domänen dargestellt. Die Frage dabei lautete stets: „Wie hoch schätzen Sie Ihre Kompetenzen in den folgenden Bereichen ein?“ 


\begin{tabular}{|c|c|c|c|c|c|c|}
\hline Item & sehr hoch & hoch & $\begin{array}{c}\text { durch- } \\
\text { schnittlich }\end{array}$ & niedrig & $\begin{array}{l}\text { nicht } \\
\text { vorhanden }\end{array}$ & $\begin{array}{l}\text { keine } \\
\text { Antwort }\end{array}$ \\
\hline $\begin{array}{l}\text { Analyse und Beurteilung } \\
\text { des eigenen } \\
\text { Chemieunterrichts }\end{array}$ & 0 & 0 & 0 & 0 & 0 & 0 \\
\hline $\begin{array}{l}\text { Auseinandersetzung mit } \\
\text { der aktuellen } \\
\text { fachdidaktischen Literatur }\end{array}$ & 0 & 0 & 0 & 0 & 0 & 0 \\
\hline $\begin{array}{l}\text { Kooperation mit } \\
\text { KollegInnen aus dem } \\
\text { Fachbereich Chemie }\end{array}$ & 0 & 0 & 0 & 0 & 0 & 0 \\
\hline $\begin{array}{l}\text { Binnendifferenzierung im } \\
\text { Chemieunterricht }\end{array}$ & 0 & 0 & 0 & 0 & 0 & 0 \\
\hline Einsatz von Experimenten & 0 & $\mathrm{o}$ & 0 & 0 & $\mathrm{o}$ & 0 \\
\hline
\end{tabular}

Tabelle 1: Beispielitems aus allen fünf Domänen

In weiterer Folge werden die Items den fünf Domänen des EPIK-Modells zugewiesen, um die Professionalität von Lehrpersonen gut abzubilden und schließlich in Abhängigkeit von Dienstalter, biologischem Alter oder Fortbildungstätigkeit untersucht zu werden. Die Kompetenzbereiche wurden mit vier bis 13 Items abgebildet.

\subsection{Datenerhebung}

Die Stichprobe ergab sich aus den im Dienst stehenden Mitgliedern des Verbandes der Chemielehrer*innen Österreichs (VCÖ). Dieser ist mit 3500 Mitgliedern der größte unpolitische Lehrendenverband Österreichs. Im März 2021 wurde Fragebogen als Limesurvey-Link über den E-Mail-Verteiler des Verbands an rund 1800 infrage kommende Lehrpersonen geschickt. Lehrende in Ausbildung oder im Ruhestand wurden ebenso ausgeschlossen wie fachfremdunterrichtende Lehrpersonen. Die Befragung endete nach zwei Wochen planmäßig mit 189 vollständig ausgefüllten Fragebögen. Dies entspricht einer Rücklaufquote von 11,79 \%, was Bortz \& Döring (2006) folgend ein zu erwartender Wert ist.

Im Anschluss an die Datenerhebung erfolgte die Datenaufbereitung mit SPSS für die statistische Hypothesenprüfung. Die Items, welche im Fragebogen einer Domäne zugeordnet wurden, wurden nun auch in SPSS zu Skalen zusammengefasst, um so das jeweilige Konstrukt bzw. die jeweilige Domäne abzubilden. Im Zuge dessen wurde auch eine Reliabilitätsprüfung für die einzelnen Skalen durchgeführt, um Items zu identifizieren, welche das Konstrukt nicht oder unbefriedigend abbilden. Der Cronbach-Alpha-Koeffizient lag bei allen Skalen über .700. Alle Skalen können daher verwendet werden (Blanz, 2015).

\subsection{Deskriptive Auswertung}

Insgesamt wurden 189 Fragebögen vollständig ausgefüllt. Von diesen Personen gaben $67(35,45 \%)$ als Geschlecht männlich und 121 (64,02 \%) weiblich an. Es nahmen somit ungefähr doppelt so viele Frauen wie Männer an der Studie teil. Ein Umstand, der angesichts der Geschlechterverteilung im Lehrberuf nicht überrascht. Eine Person bezeichnete sich als divers $(0,53 \%)$. Die Person wurde selbstverständlich bei der Hypothesenprüfung weitestgehend berücksichtigt. Einzig bei der Frage nach dem Geschlecht und seinem Einfluss auf die professionelle Selbstwahrnehmung wurde diese Lehrperson aufgrund ihres Alleinstellungsmerkmals nicht einbezogen.

Ein weiteres deskriptives Item bezog sich auf die Lehramtsausbildung, auf Basis derer die Lehrenden unterrichten. Die Proband*innen wurden gebeten, den höchsten persönlichen Lehramtsabschluss anzugeben. 107 Personen $(56,02 \%)$ gaben an, auf Basis eines universitären Lehramtstudiums zu unterrichten. Weitere 38 Lehrende (19,90 \%) absolvierten eine Pädagogische Hochschule und 44 (23,04 \%) eine Pädagogische Akademie. 
Von einer detaillierten Beschreibung der PädagogInnenbildung NEU wird aufgrund des Untersuchungsdesigns abgesehen. Diese wurde 2015 eingeführt. Angesichts einer Mindeststudiendauer von sechs Jahren bis zum Masterabschluss und der daran anschließenden einjährigen Induktionsphase können Absolvent*innen im Jahr 2021 nicht sinnvoll abgebildet werden. Zählte man die PH- und Pädak-Absolvent*innen zusammen, ergeben sich 82 Personen. Dem standen 107 Personen mit universitärem Lehramt gegenüber. Dies konnte als akzeptable Gleichverteilung betrachtet werden.

Wenngleich sich Hubermans Laufbahnmodell (1991) auf das Dienstalter anstelle des biologischen Alters bezieht und Letzteres für die Hypothesenprüfung keine Rolle spielt, darf an dieser Stelle auf die Altersverteilung der Teilnehmer*innen hingewiesen werden. Die jüngste Lehrperson war zum Zeitpunkt der Erhebung 25, die älteste 64 Jahre alt. Dazwischen gab es zumindest eine Person jeden Alters. 13 Personen waren 45 Jahre alt. Damit bildeten sie die relative Mehrheit. Der Mittelwert lag bei 42.96 Jahren $(S D=10.35)$.

Für Hubermans Modell (1991) viel relevanter ist das Dienstalter. Die teilnehmenden Lehrenden trugen die Anzahl der abgeschlossenen Dienstjahre als Zahl ein. Entsprechend den von Huberman gewählten Kategorien wurde das Item umkodiert. Eine Übersicht liefert Tabelle 2. Die dienstjüngste Person befand sich zum Zeitpunkt der Erhebung im ersten Dienstjahr (daher 0 abgeschlossene Dienstjahre). Die dienstälteste Person hat bereits 41 Jahre als Lehrperson absolviert. Der Mittelwert lag hier bei 15.82 Jahren $(S D=11.33)$.

\begin{tabular}{lll} 
Abgeschlossene Dienstjahre & Anzahl & Prozent \\
\hline 0 - 3 Jahre & 23 & 12,16 \\
4 - 6 Jahre & 31 & 16,40 \\
7 - 18 Jahre & 69 & 36,51 \\
19 - 30 Jahre & 37 & 19,58 \\
$31-45$ Jahre & 29 & 15,34 \\
\hline Gesamt & 189 & 100,00 \\
\hline
\end{tabular}

Tabelle 2: Das Dienstalter in den Kategorien von Huberman (1991)

Ein weiteres auch für die Hypothesenprüfung relevantes Item ist jenes der Fortbildungstätigkeit. Die Teilnehmer*innen wurden um eine Einschätzung gebeten, wie viele Stunden sie in einem durchschnittlichen Schuljahr absolvieren, wobei bei diesem Item keine Zahl einzutragen war, sondern eine Kategorie zu wählen war. Dies wird damit begründet, dass die Lehrpersonen hier lediglich eine grobe Schätzung abgeben und der Unterschied zwischen beispielsweise 26 oder 27 absolvierten Stunden relativ beliebig hätte ausfallen können. Mehr als die Hälfte gaben dabei an, zwischen 16 und 29 Stunden pro Jahr zu besuchen, während zehn Personen mehr als 50 Stunden pro Jahr absolvierten. In Tabelle 3 sind alle Kategorien abgebildet. Festzuhalten ist, dass für die inferenzstatistische Auswertung eine Zusammenlegung der zwei letztgenannten Kategorien erfolgte, da die Gruppengröße im Vergleich zu den anderen sehr klein war und die Kategorisierung ohnehin relativ willkürlich erfolgte.

\begin{tabular}{lll} 
Fortbildungsausma $\beta$ & Anzahl & Prozent \\
\hline Bis 15 Stunden & 61 & 32,28 \\
$16-29$ Stunden & 95 & 50,26 \\
$30-50$ Stunden & 23 & 12,17 \\
Über 50 Stunden & 10 & 5,29 \\
\hline Gesamt & 189 & 100,00 \\
\hline
\end{tabular}

Tabelle 3: Selbsteinschätzung des jährlichen Fortbildungsausmaßes

Zusammenfassend lässt sich festhalten, dass die Stichprobe in Hinblick auf Ausbildung, Schultyp, biologisches Alter, Dienstalter und Fortbildungsausmaß breit gestreut war. Zwar nahmen beispielsweise mehr Lehrende mit universitärem Lehramtsstudium an der Erhebung teil, die Variation war jedoch akzeptabel. Auch die beobachtete Geschlechterverteilung war angesichts jener im Lehrkörper generell zu erwarten. Zwar wählten einzelne Teilnehmende bei manchen Fragen die Option „keine Antwort“, jedoch ließ sich hier kein Muster erkennen. Die 
189 Fragebögen, welche von Anfang bis Ende bearbeitet wurden, bilden daher den Datensatz für die weitere Analyse.

Exemplarisch wird an dieser Stelle anhand einer Domäne die weitere Vorgehensweise demonstriert. Die erste Domäne des EPIK-Modells, Reflexions- und Diskursfähigkeit, wurde mit vier Items abgebildet. Die Antwortverteilung der einzelnen Fragen kann Tabelle 4 entnommen werden. Die teilnehmenden Lehrpersonen stufen ihre Kompetenzen relativ hoch ein. Nur bei einem Item, der Beurteilung fremder Lehrleistung, gaben fünf Personen an, hier keine Fertigkeiten zu besitzen.

\begin{tabular}{|c|c|c|c|c|c|c|}
\hline Item & sehr hoch & hoch & durchschnittlich & niedrig & $\begin{array}{c}\text { nicht } \\
\text { vorhanden }\end{array}$ & $\begin{array}{c}\text { keine } \\
\text { Antwort }\end{array}$ \\
\hline $\begin{array}{l}\text { Analyse und Beurteilung } \\
\text { des eigenen } \\
\text { Chemieunterrichts }\end{array}$ & $\begin{array}{c}25 \\
(13,2 \%)\end{array}$ & $\begin{array}{c}103 \\
(54,5 \%)\end{array}$ & $\begin{array}{c}57 \\
(30,2 \%)\end{array}$ & $\begin{array}{c}4 \\
(2,1 \%)\end{array}$ & $\begin{array}{c}0 \\
(0 \%)\end{array}$ & $\begin{array}{c}0 \\
(0 \%)\end{array}$ \\
\hline $\begin{array}{l}\text { Beurteilung von eigener } \\
\text { Lehrleistung und } \\
\text { Unterrichtsqualität }\end{array}$ & $\begin{array}{c}31 \\
(16,4 \%)\end{array}$ & $\begin{array}{c}99 \\
(52,4 \%)\end{array}$ & $\begin{array}{c}56 \\
(29,6 \%)\end{array}$ & $\begin{array}{c}3 \\
(1,6 \%)\end{array}$ & $\begin{array}{c}0 \\
(0 \%)\end{array}$ & $\begin{array}{c}0 \\
(0 \%)\end{array}$ \\
\hline $\begin{array}{l}\text { Beurteilung von } \\
\text { fremder Lehrleistung } \\
\text { und Unterrichtsqualität }\end{array}$ & $\begin{array}{c}15 \\
(7,9 \%)\end{array}$ & $\begin{array}{c}81 \\
(42,9 \%)\end{array}$ & $\begin{array}{c}74 \\
(39,2 \%)\end{array}$ & $\begin{array}{c}7 \\
(3,7 \%)\end{array}$ & $\begin{array}{c}5 \\
(2,6 \%)\end{array}$ & $\begin{array}{c}7 \\
(3,7 \%)\end{array}$ \\
\hline $\begin{array}{l}\text { Gesprächsführung im } \\
\text { Unterricht }\end{array}$ & $\begin{array}{c}50 \\
(26,5 \%)\end{array}$ & $\begin{array}{c}88 \\
(46,6 \%)\end{array}$ & $\begin{array}{c}48 \\
(25,4 \%)\end{array}$ & $\begin{array}{c}2 \\
(1,1 \%)\end{array}$ & $\begin{array}{c}0 \\
(0 \%)\end{array}$ & $\begin{array}{c}1 \\
(0,5 \%)\end{array}$ \\
\hline
\end{tabular}

Tabelle 4: Die Items der Skala Reflexions- und Diskursfähigkeit

In Tabelle 5 werden sämtliche Nennungen in absoluten Zahlen innerhalb der Skala zusammengerechnet dargestellt. Dadurch soll ersichtlich werden, wie oft sehr hoch, hoch usw. insgesamt angegeben wurden.

\begin{tabular}{lcccccc} 
Skala & sehrhoch & hoch & durchschnittlich & niedrig & $\begin{array}{c}\text { nicht } \\
\text { vorhanden }\end{array}$ & $\begin{array}{c}\text { keine } \\
\text { Antwort }\end{array}$ \\
\hline $\begin{array}{l}\text { Reflexions- und } \\
\text { Diskursfähigkeit }\end{array}$ & 121 & 371 & 235 & 16 & 5 & 8 \\
\hline
\end{tabular}

Tabelle 5: Antwortverteilung aller Items der Skala Reflexions- und Diskursfähigkeit

Für jede Skala wurden abschließend der Mittelwert sowie die Standardabweichung berechnet. Diese Werte können der Tabelle 6 entnommen werden. Dabei zeigt sich, dass der Kompetenzbereich Reflexions- und Diskursfähigkeit den niedrigsten und damit besten Mittelwert vorwies. Am schlechtesten schätzten sich global betrachtet die Lehrpersonen im Bereich Professionsbewusstsein ein. 


\begin{tabular}{lcc} 
Skala & $M$ & $S D$ \\
\hline Reflexions- und Diskursfähigkeit & 2.21 & 0.54 \\
\hline Professionsbewusstsein & 2.46 & 0.49 \\
\hline Kollegialität und Kooperation & 2.45 & 0.70 \\
\hline Differenzfähigkeit & 2.45 & 0.49 \\
\hline Personal Mastery & & \\
\hline
\end{tabular}

Tabelle 6: Der Mittelwert und die Standardabweichung für die fünf Skalen

Damit war die deskriptive Datenanalyse abgeschlossen. In weiterer Folge wurden noch die Voraussetzungen für eine multivariate Varianzanalyse geprüft, wobei ein Augenmerk auf die Suche nach extremen Ausreißern, die Prüfung auf Normalverteilung mit dem Shapiro-Wilk-Test sowie die Prüfung auf Multikollinearität gelegt wurde. Die Ergebnisse erlaubten den Beginn der inferenzstatistischen Auswertung.

\subsection{Inferenzstatistische Auswertung}

Um die Forschungsfrage zu beantworten bzw. die Hypothesen zu prüfen, wurde eine multivariate Varianzanalyse (MANOVA) durchgeführt. Die abhängigen Variablen wurden in dieser Arbeit durch die Skalen zu den einzelnen Domänen gebildet. Die Faktoren sind das Dienstalter, die Ausbildung, die Fortbildungstätigkeit und das Geschlecht.

Die Varianzanalyse ergab für den Faktor Dienstalter im Hinblick auf den multivariaten Haupteffekt einen statistisch signifikanten Unterschied mit kleiner Effektgröße in der generellen professionellen Selbstwahrnehmung, also über alle fünf Domänen gehend, zwischen Lehrpersonen mit mehr oder weniger Dienstjahren, $F(20,597)=1.695, p=.030, \eta^{2}=.045$. Bezogen auf die einzelnen Variablen konnte gezeigt werden, woraus sich der multivariate Haupteffekt zusammensetzt. Signifikante Effekte ergaben sich bei den Domänen Reflexions- und Diskursfähigkeit, $F(4,184)=3.588, p=.008, \eta^{2}=.072$, Professionsbewusstsein, $F(4,184)=4.236$, $p=.003, \eta^{2}=.084$, und Personal Mastery, $F(4,184)=3.076, p=.018, \eta^{2}=.063$. Die Effektgrößen liegen dabei immer im Bereich eines mittelgradigen Effekts (Lenhard \& Lenhard, 2016). Die Domäne Kollegialität und Kooperation, $F(4,184)=1.421, p=.229, \eta^{2}=030$, führte ebenso wenig zu signifikanten Ergebnissen wie die Differenzfähigkeit, $F(4,184)=1.323, p=.263, \eta^{2}=.028$.

Generell ließ sich durch die MANOVA somit ein kleiner, aber signifikanter Effekt für den Faktor Dienstalter beobachten. Bei der Domäne Reflexions- und Diskursfähigkeit zeigte der Mittelwertvergleich: Je länger eine Lehrperson im Dienst steht, desto höher schätzt sie ihre Fertigkeiten in diesem Bereich ein. Vor allem der Unterschied zwischen den Lehrenden mit 7 bis 18 und jenen mit 19 bis 30 Dienstjahren war signifikant. Dies ist insofern konsistent mit der Literatur, als eine Lehrperson bei Huberman (1991) mit 18 Dienstjahren entweder den Weg in die Gelassenheit oder den Konservativismus einschlägt. Beim Professionsbewusstsein ergibt sich ein ähnliches Bild. Weist ein*e Lehrer*in mehr Dienstjahre auf, werden die eigenen Kompetenzen höher eingeschätzt. Dies ist nicht überraschend, da in dieser Domäne Items enthalten waren, welche besonders stark erfahrungsabhängig sind (z. B. Zeitmanagement, Leistungsbeurteilung, Verfassen von Planungen). Auch die Personal Mastery hängt, zumindest was die subjektive Wahrnehmung betrifft, vom Dienstalter ab. Dienstältere Chemielehrende nehmen sich signifikant als besser wahr als dienstjüngere. Dies hängt womöglich damit zusammen, dass erfahrene Lehrende auf ein großes Repertoire an Experimenten zurückgreifen können, sich weniger Gedanken bezüglich fachlicher Richtigkeit ihrer Lehrinhalte machen oder auf Basis ihrer Erfahrung einschätzen können, wo das Unterrichtsniveau anzusetzen ist. Auch hier decken sich Hubermans (1991) Überlegungen und die empirischen Ergebnisse. Die Personal Mastery wird von Berufsneulingen, welche gerade den Berufseinstieg überstehen müssen, und Lehrenden im letzten Abschnitt ihrer Laufbahn, welche Huberman (1995) als Disengagement bezeichnet, am niedrigsten eingestuft. Da bei Letzterem der Schwerpunkt auf das „Ausklingen der beruflichen Tätigkeit“ (S. 249) gelegt wird, sind die empirischen Befunde stimmig. Das 
Dienstalter scheint beim Umgang mit Diversität keine bedeutende Rolle zu spielen. In dieser Domäne findet sich also das Modell von Huberman (1991) nicht wieder.

Im Einklang mit der Literatur war ferner der Umstand, dass sich Lehrende in der ersten Phase ihrer Tätigkeit, in der sie Huberman (1991) zufolge vor allem mit dem „Überleben“ im Klassenzimmer beschäftigt sind, beinahe in allen Domänen am schlechtesten im Vergleich zu den Lehrpersonen mit mehr Erfahrung einstufen. Wenngleich dies lediglich bei der Domäne Personal Mastery signifikant war, ließ sich dennoch eine Tendenz bei allen Domänen erkennen. Zusammenfassend kann festgehalten werden, dass die Hypothese, wonach das professionelle Selbstempfinden von Lehrpersonen unterschiedlicher Diensterfahrung divergiert, bestätigt werden konnte. Die Nullhypothese konnte verworfen werden. Es darf jedoch auf die kleine Effektgröße verwiesen werden $\left(\eta^{2}=.045\right)$.

Die Varianzanalyse ergab mit Blick auf den multivariaten Haupteffekt keinen statistisch signifikanten Unterschied hinsichtlich der professionellen Selbstwahrnehmung zwischen Lehrpersonen mit einem Lehramts-, $\mathrm{PH}$ - oder Pädak-Studium, $F(10,364)=1.480, p=.145, \eta^{2}=.039$. Bei den einzelnen Variablen ergaben sich dadurch auch keine signifikanten Differenzen. Weder bei der Reflexions- und Diskursfähigkeit, $F(2,186)=0.472, p=.625$, $\eta^{2}=.005$, dem Professionsbewusstsein, $F(2,186)=0.199, p=.820, \eta^{2}=.002$; der Kollegialität und Kooperation, $F(2,186)=1.445, p=.238, \eta^{2}=.015$, noch der Differenzfähigkeit, $F(2,186)=1.024, p=.361, \eta^{2}=.011$, oder der Personal Mastery, $F(2,186)=0.796, p=.453, \eta^{2}=.008$, ergaben sich signifikante Unterschiede im Professionsempfinden zwischen den Absolvent*innen der verschiedenen Ausbildungsvarianten. Dementsprechend zeigte auch der Post-hoc-Test nach Bonferroni keinerlei Signifikanzen.

Dies darf durchaus als überraschend betrachtet werden. Bei den Domänen Reflexions- und Diskursfähigkeit sowie Personal Mastery ließ sich eine Tendenz erkennen, wonach Personen mit universitärem Lehramtsstudium $(M=2.19, S D=0.49$ bzw. $M=2.20, S D=0.43)$ ihre Fertigkeiten höher einschätzen als PädakAbsolvent*innen $(M=2.28, S D=0.67$ bzw. $M=2.31, S D=0.45)$. Bei beiden Domänen liegen Lehrende mit PHAusbildung zwischen diesen beiden Gruppen $(M=2.22, S D=0.53$ bzw. $M=2.24, S D=0.51)$. Bei Kollegialität und Kooperation $(M=2.33, S D=0.71)$ sowie Differenzfähigkeit $(M=2.35, S D=0.53)$ schätzten sich $\mathrm{PH}$ Absolvent*innen am besten von den drei Gruppen ein und beim Professionsbewusstsein $(M=2.50, S D=0.55)$ am schlechtesten. Es darf aber angemerkt werden, dass die Mittelwertunterschiede verschwindend gering sind. Die Form der Ausbildung scheint somit eine untergeordnete Rolle bei der Selbsteinschätzung zu spielen. Bei der Begutachtung der Standardabweichung ergab sich ebenso wenig ein eindeutiges Bild. Zwar gingen die Werte für die Domäne Reflexions- und Diskursfähigkeit bei Menschen mit Pädak-Ausbildung deutlich mehr auseinander als bei jenen mit Lehramtsstudium, aber schon bei der Personal Mastery zeigte sich ein umgekehrtes Bild. Da die inferenzstatistische Auswertung keinerlei signifikante Ergebnisse lieferte, kommt der Autor zum Schluss, dass die Hypothese, wonach es Unterschiede im Professionsempfinden zwischen den Absolvent*innen der einzelnen Ausbildungswege gibt, verworfen werden muss. Die Art der Ausbildung hat keinen signifikanten Einfluss darauf, ob sich eine Lehrperson als professioneller empfindet. Dies ist insofern interessant, als die Schwerpunkte der einzelnen Institutionen teilweise völlig konträr waren und sind. Auf das subjektive Empfinden scheint dies keinen Einfluss zu haben. Die Nullhypothese wird daher angenommen.

Für den Faktor Fortbildung konnte ein signifikanter Unterschied zwischen den drei Gruppen (unter 15 Stunden, 16-29 Stunden und über 30 Stunden) im Hinblick auf das Professionsempfinden festgestellt werden, $F(10,364)=2.793, p=.002, \eta^{2}=.070$. Bezogen auf die einzelnen Variablen wurde erneut untersucht, woraus sich der multivariate Haupteffekt zusammensetzt. Signifikante Differenzen ergaben sich bei den Domänen Kollegialität und Kooperation, $F(2,186)=8.652, p<.001, \eta^{2}=.085$, Differenzfähigkeit, $F(2,186)=6.684, p=.002$, $\eta^{2}=.067$, und Personal Mastery, $F(2,186)=7.272, p=.001, \eta^{2}=.073$. Die Effektgrößen liegen damit im mittelgradigen Bereich (Lenhard \& Lenhard, 2016). Beim Professionsbewusstsein ergab sich knapp kein signifikantes Ergebnis, $F(2,186)=2.884, p=.058, \eta^{2}=.030$. Der Unterschied zwischen den einzelnen Gruppen bei der Reflexions- und Diskursfähigkeit war eindeutig nicht signifikant, $F(2,186)=1.324, p=.269, \eta^{2}=.014$. Die Hypothese, wonach es einen Unterschied im Professionsempfinden zwischen jenen mit hoher und jenen mit niedriger Fortbildungstätigkeit gibt, konnte bestätigt werden, wenngleich auch hier trotz Signifikanz auf die mittelgradige Effektgröße verwiesen werden muss. Lehrpersonen mit mehr als 30 Fortbildungsstunden pro Jahr empfanden sich signifikant als professioneller in den Bereichen Kollegialität und Kooperation, Differenzfähigkeit und Personal Mastery. Dies ist nicht überraschend, sind es doch vor allem die Bereiche Differenzfähigkeit und Personal Mastery, welche in den meisten Fortbildungsveranstaltungen zum Thema gemacht werden. So werden neue didaktische Zugänge, Experimente und Möglichkeiten zur Differenzierung besonders häufig vermittelt. Besucht eine Lehrperson viele derartige Kurse und Seminare, lernt sie auch andere Menschen aus ihrem Fachbereich kennen. Eine stärkere Vernetzung und damit höhere Kooperation können die Folge sein. 
Nicht nur die inferenzstatistische Auswertung, auch die Mittelwertvergleiche zeigen ein einheitliches, wenngleich auch nicht immer statistisch signifikantes Bild. Lehrende in der Gruppe mit den meisten Fortbildungsstunden stufen sich in allen Domänen besser ein als jene, die nur bis zu 15 Stunden pro Jahr besuchen. Dies kann als Zeichen für eine funktionierende Lehrendenfortbildung interpretiert werden. Dass es zwischen der Gruppe mit 16 bis 29 und jener mit über 30 Stunden keine signifikanten Unterschiede gab, lässt sich möglicherweise damit erklären, dass Landeslehrpersonen 15 Stunden verpflichtende Fortbildungen zu absolvieren haben und die Gruppe mit bis zu 15 Stunden pro Jahr nur ihre dienstliche Pflicht erfüllt. Die anderen beiden Gruppen gehen darüber hinaus. Dies berücksichtigend könnte man die drei Gruppen nochmals unterteilen in jene, die ihre dienstliche Fortbildungspflicht erfüllen, und jene, die darüber hinausgehen.

Durch die MANOVA konnte abschließend ein signifikanter Unterschied bei der professionellen Selbstwahrnehmung zwischen männlichen und weiblichen Lehrpersonen festgestellt werden, $F(5,182)=4.463$, $p=.001, \eta^{2}=.109$. Die Effektgröße entspricht einer gehobenen mittelgradigen Effektgröße (Lenhard \& Lenhard, 2016). Bei der Untersuchung der einzelnen Variablen konnte errechnet werden, dass diese Signifikanz auf eine einzige Variable zurückgeht. Lediglich bei der Domäne Differenzfähigkeit gibt es ein statistisch signifikantes Ergebnis, $F(1,186)=4.803, p=.030, \eta^{2}=.025$. Die Unterschiede in den Domänen Reflexions- und Diskursfähigkeit, $F(1,186)=.348, p=.557, \eta^{2}=.002$, Professionsbewusstsein, $F(1,186)=.239, p=.625, \eta^{2}=.001$; Kollegialität und Kooperation, $F(1,186)=.657, p=.419, \eta^{2}=.004$, und Personal Mastery, $F(1,186)=1.491, p=$ $.224, \eta^{2}=.008$, sind alle statistisch nicht signifikant. Da der Faktor Geschlecht in dieser Kalkulation nur aus zwei Gruppen besteht, sind Post-hoc-Tests nicht möglich. Ein zusätzlich durchgeführter t-Test nach Pearson für unabhängige Stichproben ergab ein identes Bild. Frauen stuften sich selbst bei der Differenzfähigkeit am deutlichsten besser ein (95\%-CI[0.02, 0.31]), wodurch sich einzig bei dieser Domäne ein statistisch signifikantes Ergebnis zeigte, $t(186)=2.19, p=.030$. Auch der Mittelwertvergleich ergab, dass sich Frauen in vier der fünf Domänen tendenziell ein wenig besser einschätzten als ihre männlichen Kollegen. Einzig bei der Personal Mastery liegen die Männer $(M=2.18, S D=0.46)$ bei der Selbsteinschätzung leicht vor den Frauen $(M=2.26$, $S D=0.44)$. Der größte Unterschied ergab sich bei der Domäne Differenzfähigkeit, wo sich Frauen $(M=2.39$, $S D=0.51)$ am deutlichsten besser als die Männer $(M=2.55, S D=0.46)$ einschätzten. Es war durchaus überraschend, dass sich Frauen tendenziell als professioneller wahrnehmen als Männer. Das signifikante Ergebnis für die Differenzfähigkeit beruht auf Items, welche vorwiegend Soft Skills abbildeten (z. B. Sozialformen im Unterricht, Rücksichtnahme auf Lernschwierigkeiten). Im Zuge einer weiteren Untersuchung wäre es interessant, diesen Punkt aufzugreifen und zu hinterfragen, wieso gerade diese Domäne messbare Unterschiede zeigte. Ebenfalls auffällig ist der Umstand, dass sich die Männer nur in der Personal Mastery, wo es um das klassische „Unterrichten-Können“, also um Experimente und Formelsprache ging, als professioneller als die Frauen empfinden. Salopp könnte man formulieren, dass Männer überzeugter davon sind, die „Hard Facts“ zu beherrschen, als Frauen. Diese sehen ihre Stärken beim Umgang mit Diversität am stärksten ausgeprägt. An dieser Stelle darf noch ein Blick auf die Literatur geworfen werden, divergieren die theoretischen Ansätze von Sikes et al. (1991) und die empirischen Daten doch teilweise. Sikes et al. (1991) gehen in ihrem Modell davon aus, dass sich beispielsweise in der Phase von 30 bis 40 Lebensjahren weibliche Lehrende aus dem Beruf eher zurückziehen und sich Männer stärker im Beruf verwirklichen. Was auf Basis der Daten dieser Studie gesagt werden kann, ist, dass dies, sofern es tatsächlich stattfindet, keinen Einfluss auf die professionelle Selbstwahrnehmung hat. Zumindest nicht in jene Richtung, dass sich Frauen als weniger professionell empfinden. Sie stuften sich beinahe immer als professioneller ein als die Männer. Aufgrund der Ergebnisse kann der Schluss gezogen werden, dass sich Männer und Frauen hinsichtlich ihres Professionsempfindens signifikant unterscheiden. Die Alternativhypothese kann somit angenommen und die Nullhypothese verworfen werden. Eingeschränkt werden muss das Ergebnis jedoch in Hinblick auf die mittelgradige Effektstärke $\left(\eta^{2}=.109\right)$. Ein Effekt ist vorhanden, aber nur mittelstark ausgeprägt.

\section{Beantwortung der Forschungsfrage und Conclusio}

Die theoretische Grundlage für die Forschungsfrage bildete zum einen das Laufbahnmodell von Huberman (1991), zum anderen das EPIK-Modell von Schratz et al. (2007a; 2007b). Auf Basis dieser Konzepte konnte gezeigt werden, dass die Anzahl der Dienstjahre einen kleinen, aber signifikanten Effekt auf die subjektiv wahrgenommene Professionalität einer Chemielehrperson hat. Lehrende, die länger im Dienst stehen, schätzen ihre Fertigkeiten im Bereich der Reflexion, des Professionsbewusstseins sowie der Personal Mastery signifikant höher ein als ihre jüngeren Kolleg*innen. Bei zwei Domänen jedoch, der Kollegialität und Kooperation sowie der Differenzfähigkeit, ergaben sich keine statistisch signifikanten Unterschiede. Da die Ergebnisse der ersten drei 
Domänen gegenüber den anderen beiden überwiegen, kann die leitende Forschungsfrage positiv beantwortet werden.

Die Ergebnisse, wonach die Ausbildungsvariante keinen Einfluss auf das Professionsempfinden zu haben scheint, waren für den Autor überraschend. Ob der Ausbildungsschwerpunkt also auf die Fachwissenschaft oder die Didaktik bzw. Humanwissenschaften gelegt wird, hat keinen Einfluss darauf, als wie professionell sich eine Lehrperson wahrnimmt. Hier wurde die Nullhypothese angenommen, denn keine einzige Signifikanz konnte nachgewiesen werden. Dieser Aspekt verlangt wohl nach einem anderen Testformat wie beispielsweise einem Performanztest.

Die Rolle der Fortbildung und Weiterbildung hingegen war eindeutig. Es gibt einen Unterschied hinsichtlich der subjektiv wahrgenommenen Professionalität zwischen Lehrpersonen mit einer hohen und solchen mit einer geringen Fortbildungstätigkeit. In drei von fünf Domänen steigt das Professionsempfinden signifikant mit steigender Fortbildungstätigkeit an. Die Alternativhypothese konnte angenommen werden. Dies kann als Auftrag für den Dienstgeber sowie die Pädagogischen Hochschulen verstanden werden, welche die Fortbildungen in Österreich koordinieren. Bilden sich Chemielehrende häufiger fort, steigen ihre selbst empfundene Personal Mastery, die Fähigkeit, mit Differenzen im Unterricht umzugehen, sowie das Können, mit anderen in und außerhalb der Schule zusammenzuarbeiten, was, wie in dieser Arbeit mehrfach gezeigt wurde, eine Grundbedingung in der Schule des 21. Jahrhunderts ist.

Dass sich weibliche und männliche Lehrpersonen nur geringfügig in ihrem Professionsempfinden unterscheiden, war etwas überraschend. Klischeehafte und vorurteilsbeladene Vorannahmen hatten vermuten lassen, dass sich Männer generell als professioneller einschätzen. Es sollte sich jedoch herausstellen, dass sich Lehrer nur bei den „Hard Facts“, also der Personal Mastery, als besser einschätzen, wobei hier nochmals darauf verwiesen werden soll, dass die Ergebnisse nicht signifikant waren. Vielmehr konnten nur leichte Tendenzen herausgelesen werden. Einzig beim Umgang mit Unterschieden im Unterricht zeigt sich ein tatsächlich signifikanter Unterschied zwischen den zwei untersuchten Geschlechtern.

Zusammenfassend kann festgehalten werden, dass das Professionsempfinden von zwei der vier untersuchten Faktoren abhängt. Die Größe des Einflusses von Dienstalter und Fortbildung darf jedoch nicht überinterpretiert werden.

Die Arbeit bietet eine Reihe von forschungsbezogenen Anknüpfungspunkten. So wäre es aus Sicht der Berufspraxis relevant, ungeprüfte Chemielehrer*innen hinsichtlich ihres Professionsempfindens zu befragen. Ferner könnte man den Forschungsschwerpunkt auf die Volksschule legen und untersuchen, als wie professionell sich Primarstufenlehrende im Bereich des chemischen Erstunterrichts empfinden, was wiederum Konsequenzen für die Aus-, Fort- und Weiterbildung dieses Personenkreises haben könnte. Ferner darf an dieser Stelle darauf hingewiesen werden, dass nicht erhoben wurde, wie professionell Fachlehrende sind, sondern wie professionell sie sich fühlen. Auch dies eröffnet interessante Möglichkeiten für weitere Forschungsvorhaben. So könnte man mithilfe eines Performanztests messen, wie professionell Chemielehrende tatsächlich in Abhängigkeit von Dienstalter, Geschlecht, Fortbildungstätigkeit oder Ausbildung sind, und die Ergebnisse in Relation zum persönlichen Empfinden setzen.

Am Ende dieser Arbeit steht somit eine Reihe von weiteren Fragen. Insofern schließt sich wieder der Kreis zu den ursprünglichen Überlegungen im theoretischen Teil der Studie. Genauso wenig, wie man als einzelne Lehrperson jemals wirklich „fertig“ ist, sind die Überlegungen zur Profession der Lehrenden im Allgemeinen jemals wirklich abgeschlossen.

\section{Literatur}

Bastian, J., \& Helsper W. (2000). Professionalisierung im Lehrberuf: Bilanzierung und Perspektiven. In J. Bastian, W. Helsper, S. Reh, \& C. Schelle (Hrsg.), Professionalisierung im Lehrberuf: Von der Kritik der Lehrerrolle zur pädagogischen Professionalität (S. 167-192). Leske + Budrich.

Bauer, K.-O. (2005). Pädagogische Basiskompetenzen. Theorie und Training. München: Juventa.

Behr, F. (2017). Lernhabitus und Weiterentwicklung. Klinkhard.

Blanz, M. (2015). Forschungsmethoden und Statistik für die Soziale Arbeit: Grundlagen und Anwendungen. Kohlhammer.

Bortz, J., \& Döring, N. (2006). Forschungsmethoden und Evaluation für Human- und Sozialwissenschaftler (4. Aufl.). Springer. 
R\&E-SOURCE $h$ ttps://journal.ph-noe.ac.at Online Journal for Research and Education

Ausgabe 16, Oktober 2021, ISSN: 2313-1640

Dammerer, J. (2020). Berufszufriedenheit und lebenslanges Lernen von Lehrpersonen in der niederösterreichischen Volksschule. Dissertation. Alpen-Adria-Universität Klagenfurt.

Dammerer, J. \& Schwab, K. (2019). Entwicklungsmodelle von beginnenden Lehrpersonen: Eine vergleichende qualitative Untersuchung zum Phasenmodell nach Huberman und Stufenmodell nach Fuller \& Brown. Online Journal for Research and Education, 12.

Dreyfus, H. L., \& Dreyfus, S. E. (1988). Künstliche Intelligenz: Von den Grenzen der Denkmaschine und dem Wert der Intuition. Rowohlt.

Eilks, I., \& Marks, R. (2018). Chemielernen für gesellschaftliche Teilhabe und nachhaltige Entwicklung. In V. Reinhardt, M. Rehm \& M. Wilhelm (Hrsg.), Wirksamer Chemieunterricht: Unterrichtsqualität Perspektiven von Expertinnen und Experten (S. 37-47). Schneider.

Hericks, U. (2006). Professionalisierung als Entwicklungsaufgabe. Wiesbaden: Verlag für Sozialwissenschaften.

Herrmann, U., \& Hertramph, H. (2000). Zufallsroutinen oder reflektierte Praxis? Herkömmliche Wege in den Berufseinstieg von Lehrern und notwendige Alternativen. Beiträge zur Lehrerinnen- und Lehrerbildung, 18 (2), 172-191.

Hilfert-Rüppell, D., Eghtessad, A., Looß, M., \& Höner, K. (2012). Empirische Studien zum Professionalisierungsprozess in den naturwissenschaftlichen Fächern der Lehramtsstudiengänge. Lehrerbildung auf dem Prüfstand, 5 (2), 157-179.

Huberman, M. (1991). Der berufliche Lebenszyklus von Lehrern: Ergebnisse einer empirischen Untersuchung. In E. Terhart (Hrsg.), Unterrichten als Beruf: Neue amerikanische und englische Arbeiten zur Berufskultur und Berufsbiographie von Lehrern und Lehrerinnen (S. 249-267). Böhlau.

Klusmann, U., Kunter, M., Voss, T., \& Baumert J. (2012). Berufliche Beanspruchung angehender Lehrkräfte: Die Effekte von Persönlichkeit, pädagogischer Vorerfahrung und professioneller Kompetenz. Zeitschrift für Pädagogische Psychologie, 26 (4), S. 275-290.

Kraler, C. (2008). Professionalisierung in der Berufseingangsphase - Berufsbiografie und Kompetenzentwicklung: Entwicklungsaufgaben der ersten Berufsjahre und Unterstützungsmöglichkeiten. SchulVerwaltung spezial: Zeitschrift für Schulgestaltung und Schulentwicklung, 1, S. 4-7.

Lenhard, W. \& Lenhard, A. (2016). Berechnung von Effektstärken. Statistische Beratung Linda Regber. http://lindaregber.com/effekt-berechnen-interpretieren

Messner, H. \& Reusser, K. (2000). Die berufliche Entwicklung von Lehrpersonen als lebenslanger Prozess. Beiträge zur Lehrerbildung, 18 (2), 157-171.

Paseka, A., Schratz, M., \& Schrittesser, I. (2011). Professionstheoretische Grundlagen und thematische Annäherung. In A. Paseka, M. Schratz \& I. Schrittesser (Hrsg.), Pädagogische Professionalität: quer denken - umdenken - neu denken: Impulse für next practice im Lehrberuf (S. 8-45). Facultas.

Rehm, M. (2018). Wirksamer Chemieunterricht: Eine Einführung. In V. Reinhardt, M. Rehm \& M. Wilhelm (Hrsg.), Wirksamer Chemieunterricht: Unterrichtsqualität - Perspektiven von Expertinnen und Experten (S. 17-24). Schneider.

Schmidt, J., Klusmann, U., \& Kunter, M. (2016). Wird alles besser? Positive und negative berufliche Ereignisse von Referendarinnen bzw. Referendaren und Lehrkräften im Vergleich. Psychologie in Erziehung und Unterricht, 63, S. 278-291.

Schratz, M., Schrittesser, I., Forthuber, P., Pahr, G., Paseka, A., \& Seel, A. (2007a). Domänen von Lehrer/innen/professionalität: Entwicklung von Professionalität im internationalen Kontext (EPIK). Journal für Lehrerinnen- und Lehrerbildung, 7, 70-80.

Schratz, M., Schrittesser, I., Forthuber, P., Pahr, G., Paseka, A., \& Seel, A. (2007b). Domänen von Lehrer/innen/professionalität: Rahmen einer kompetenzorientierten Lehrer/innen/bildung. In C. Kraler \& M. Schratz (Hrsg.), Wissen erwerben, Kompetenzen entwickeln: Modelle zur kompetenzorientierten Lehrerbildung (S. 123-138). Waxmann.

Schratz, M. \& Schrittesser I. (2011). Was müssen Lehrerinnen und Lehrer in Zukunft wissen und können? In H. Berner \& R. Isler (Hrsg.), Lehrer-Identität-Lehrer-Rolle - Lehrer-Handeln: Professionswissen von Lehrerinnen und Lehrern (S. 177-201). Schneider.

Senge, P. M. (2006). Die fünfte Disziplin: Kunst und Praxis der lernenden Organisation (10. Aufl.). Klett-Cotta.

Sikes, P. J., Measor, L., \& Woods, P. (1991). Berufslaufbahn und Identität im Lehrberuf. In E. Terhart (Hrsg.), Unterrichten als Beruf: Neue amerikanische und englische Arbeiten zur Berufskultur und Berufsbiographie von Lehrern und Lehrerinnen (S. 231-248). Böhlau.

Stahl, U. (1995). Professionalität und Zufriedenheit im Beruf: Eine empirische Studie an Grund- und Hauptschulen. Deutscher Studienverlag. 
R\&E-SOURCE $h$ ttps://journal.ph-noe.ac.at

Online Journal for Research and Education

Ausgabe 16, Oktober 2021, ISSN: 2313-1640

Terhart, E., Czerwenka, K., Ehrich, K., Jordan, F., \& Schmidt, H. J. (1994). Berufsbiographien von Lehrern und Lehrerinnen. Lang.

Terhart, E. (2001). Lehrerberuf und Lehrerbildung: Forschungsbefunde, Problemanalysen, Reformkonzepte. Beltz.

Uhl, R.M. (2017). Professionalität in der beruflichen Bildung (Nicht veröffentlichte Dissertation). LeoploldFranzens-Universität Innsbruck. 